\title{
Prognostic nomogram for surgery of lung cancer in HIV-infected patients
}

\author{
Lin Wang ${ }^{1 \#}$, Liwei Wu ${ }^{1 \#}$, Jianjian Liu ${ }^{2 \#}$, Laiyi Wan ${ }^{1}$, Hui Chen ${ }^{1}$, Yijun Zhu ${ }^{1}$, Jun Wang ${ }^{1}$, Hongwei Li $^{1}$, \\ Lei Shi ${ }^{1}$, Leilei Li ${ }^{1}$, Yanzheng Song ${ }^{1}$ \\ ${ }^{1}$ Department of Thoracic Surgery, Shanghai Public Health Clinical Center, Fudan University Shanghai, China; ${ }^{2}$ Department of Ultrasonic Room, \\ Shanghai Public Health Clinical Center, Shanghai, China \\ Contributions: (I) Conception and design: Y Song, L Wang; (II) Administrative support: All authors; (III) Provision of study materials or patients: All \\ authors; (IV) Collection and assembly of data: All authors; (V) Data analysis and interpretation: All authors; (VI) Manuscript writing: All authors; (VII) \\ Final approval of manuscript: All authors. \\ \#These authors contributed equally to this work. \\ Correspondence to: Yanzheng Song. Department of Thoracic Surgery, Shanghai Public Health Clinical Center, Fudan University Shanghai, Caolang \\ Road 2901, Jinshan District, Shanghai, China. Email: yanzhengsong@163.com.
}

\begin{abstract}
Background: This study aimed to establish an effective prognostic nomogram for surgery of lung cancer in HIV-infected patients.

Methods: The nomogram is based on a retrospective study of 51 patients who underwent lung cancer surgery at the Shanghai Public Health Clinical Center from July 2012 to November 2019. The predictive accuracy and discriminative ability of the nomogram were determined by concordance index (C-index) and calibration curve analysis. Internal validity was assessed using bootstrapping validation.

Results: Predictors contained in the prognostic nomogram included age, CD4+ cell count, surgery method, and pathological stage. The model displayed good discrimination with a C-index of 0.755 (95\% CI: $0.715-0.795$ ) and good calibration. A high C-index value of 0.844 was reached after internal validation.
\end{abstract}

Conclusions: The proposed nomogram may result in more-accurate prognostic predictions for surgery of lung cancer in HIV-infected patients.

Keywords: Nomogram; human immunodeficiency virus; non-small cell lung cancer (NSCLC); prognosis

Submitted Jun 20, 2020. Accepted for publication Nov 04, 2020.

doi: $10.21037 /$ jtd-20-2268

View this article at: http://dx.doi.org/10.21037/jtd-20-2268

\section{Introduction}

Malignant lung tumors are often the main cause of death in patients with HIV infection (1). In the pre-antiretroviral (pre-ART) era, AIDS-defining cancers (ADCs) such as Kaposi sarcoma (KS) and non-Hodgkin's lymphoma (NHL) were common lung tumors in this group (2). With the widespread use of ART, the mortality caused by ADCs has gradually decreased. But the mortality caused by nonAIDS-defining cancers (NADCs) is gradually increasing. Malignant lung tumors, especially non-small cell lung cancer (NSCLC), have become the main cause of death in AIDS patients in the USA (3-5). followed in frequency by small-cell lung cancer. Some epidemiological studies have shown that unusual forms of lung cancer may also occur, such as lung sarcoma and neuroendocrine tumors (6).

At present, there are no guidelines for the treatment of lung cancer in patients with HIV infection. For early lung cancer, surgical treatment is generally adopted (7). Studies have shown that the prognosis of patients with HIV infection is worse than that of patients with no HIV infection (6). Nomograms are used now in a wide variety of tumor fields to predict the prognosis associated with tumor treatment (8). However, there has been no study to establish a model to predict the postoperative prognosis of lung cancer patients with HIV infection. As far as we know, 
this study is the first and is based on the clinicopathological data from 51 such patients who underwent surgery. It is expected that the prediction model can guide future clinical work. We present the following article in accordance with the TRIPOD reporting checklist (available at http://dx.doi. org/10.21037/jtd-20-2268).

\section{Methods}

\section{Patients}

For this retrospective study, we recruited patients from November 2011 to November 2019, at Shanghai Public Health Clinical Center, China which is a hospital specializing in infectious diseases. Most patients had been diagnosed as HIV-1 antibody-positive in regional hospitals and were already being treated before being referred to this center for further management. The overall survival time was calculated from the date of surgery to the time of death or the last follow-up. The follow-up was until February 1,2020 , with an average duration of $25.78 \pm 20.60$ months. The study was conducted in accordance with the Declaration of Helsinki (as revised in 2013). The study was approved by the Shanghai Public Health Clinical Center's Ethics Committee (Approval No. [2018] Y038) and written informed consent was obtained from the patients involved before enrolment, including when the data were collected retrospectively. The inclusion criteria were as follows: (I) a whole-blood confirmatory test conducted by the local CDC laboratory was positive for anti-HIV-1 antibody; (II) compliance with the TNM staging of lung cancer in the eighth edition interpretation of the International Association for the Study of Lung Cancer (IASLC); (III) post-operative pathology diagnosis of NSCLC; (IV) lung cancer clinical stages I-IIIA. The exclusion criteria were as follows: (I) mental illness leading to the patient being unable to comply with the study requirements; (II) cerebrovascular, hypertension, diabetes, and other surgical contraindications; (III) poor compliance.

After operation, the patients were followed up every two months during the first two years. Follow-up was subsequently performed every six months. Highly active antiretroviral therapy (HAART), CD4+ cell count and HIV RNA count were recorded in detail at each follow-up. The overall survival time (OS) and time to recurrence (TTR) were taken as the primary end-points. OS was defined as the interval between surgery and death or the date of a final follow-up. TTR was defined as the interval between surgery and recurrence.

\section{Statistical analysis}

Statistical analysis was performed using the $\mathrm{R}$ software (Version 3.6.2; https:// www.R-project.org). Cox regression analysis was used for multivariate analyses. The performance of the nomogram was measured by the concordance index (C-index) and calibration curves (9-11). The larger the $\mathrm{C}$-index, the more accurate was the prognostic prediction $(9,10)$. To quantify the discrimination performance of the nomogram, Harrell's C-index was measured. The nomogram was subjected to bootstrapping validation (1,000 bootstrap re-samples) to calculate a relatively corrected $\mathrm{C}$-index (12-14). $\mathrm{P}<0.05$ was considered statistically significant.

\section{Results}

\section{Patients' characteristics}

In total, 51 patients were enrolled in the study. There were 44 males and 7 females, the median age was 57 [30-77], At diagnosis, the mean serum CD4+ cell count was $395.37 \pm 188.64 / \mu \mathrm{L}$ (range, $33-940 / \mathrm{mm}^{3}$ ). Thoracotomy was performed in 27 patients $(52.9 \%)$ and video-assisted thoracoscopic surgery (VATS) in 24 patients $(47.1 \%)$. Eleven patients $(21.5 \%)$ underwent partial resection, 38 patients $(74.5 \%)$ underwent lobectomy, 2 patients $(4 \%)$ bi-lobectomy. There were 39 cases of stage I, 5 cases of stage II, 6 cases of stage IIIA, and 1 case of stage IV. The only patient with stage IV lung cancer was diagnosed as stage IIIA before the operation, and the tumor metastasis was found during the operation, so the postoperative stage was stage IV. All relevant data of patients including demographic, disease, and treatment features are given in Table 1.

\section{Independent prognostic factors in the cohort}

Multivariate analyses demonstrated that age, CD4+ cell count, surgery method, and pathology stage were independent risk factors for tumor OS (Table 2). The hazard ratios (HR) of the CD4+ cell counts were 2.801, 16.796, and 2.849. The HR of the operation type was 3.342. The remainder of the features were not statistically significant.

\section{Prognostic nomogram}

The developed model that incorporated all the independent predictors is presented as a nomogram (Figure 1). The 
Table 1 Demographic and clinicopathologic characteristics of HIVinfected patients with lung cancer

\begin{tabular}{|c|c|}
\hline Variable & Number of patients (\%) \\
\hline \multicolumn{2}{|l|}{ Age (years) } \\
\hline$<50$ & $9(17.6)$ \\
\hline $50-60$ & $24(47.1)$ \\
\hline$>60$ & $18(35.3)$ \\
\hline \multicolumn{2}{|l|}{ Gender } \\
\hline Male & 44 (86.3) \\
\hline Female & $7(13.7)$ \\
\hline \multicolumn{2}{|l|}{ HAART (months) } \\
\hline$<60$ & $38(74.5)$ \\
\hline $60-120$ & $11(21.6)$ \\
\hline$>120$ & $2(3.9)$ \\
\hline \multicolumn{2}{|l|}{ HIVRNA (copy) } \\
\hline$<40$ & $26(51)$ \\
\hline $40-475,000$ & $24(47.1)$ \\
\hline$>475,000$ & $1(2.0)$ \\
\hline \multicolumn{2}{|l|}{ CD4 cell counts } \\
\hline$<300$ & $15(29.4)$ \\
\hline $300-600$ & $30(58.8)$ \\
\hline $601-900$ & $5(9.8)$ \\
\hline$>900$ & $1(2.0)$ \\
\hline \multicolumn{2}{|l|}{ Tumor stage } \\
\hline IA & $32(62.7)$ \\
\hline IB & $7(13.7)$ \\
\hline$\| \mathrm{A}$ & $1(2.0)$ \\
\hline IIB & $4(7.8)$ \\
\hline IIIA & $6(11.8)$ \\
\hline IIIB & $0(0.0)$ \\
\hline IV & $1(2.0)$ \\
\hline \multicolumn{2}{|l|}{ Surgery method } \\
\hline Thoracotomy & $27(52.9)$ \\
\hline VATS & $24(47.1)$ \\
\hline \multicolumn{2}{|l|}{ Pathology type } \\
\hline Adenocarcinoma & $34(66.7)$ \\
\hline SCC & $9(17.6)$ \\
\hline Other & $8(15.7)$ \\
\hline
\end{tabular}

HAART, highly active antiretroviral therapy; VATS, video-assisted thoracoscopic surgery; SCC, squamous cell carcinoma. nomogram included the four features: age, CD4+ cell count, stage, and surgery method.

\section{Apparent performance of the nomogram in the cohort}

The calibration plot for the probability of survival at 3 or 5 years after surgery showed an optimal agreement between the prediction by nomogram and actual observation (Figure 2). The C-index for the prediction was 0.755 (95\% CI: $0.715-$ 0.795 ) for the cohort and was enhanced to 0.844 through bootstrapping validation, which suggested the model's good discrimination and prediction capability.

\section{Discussion}

Nowadays, nomograms are widely used in oncology and other areas of medicine (15). They provide a friendlier interface, easier to understand prognosis, and higher accuracy, which can help clinical decision-making $(16,17)$. In our study, for the first time a nomogram was developed to predict the prognosis of lung cancer in patients with HIV infection. The validated new predictive tool used only four readily available variables. The incorporation of demographic, surgical, ART treatment, and other risk factors into an easy-to-use nomogram will be helpful for future individualized predictions. The internally-validated good discrimination that was found with this large number of patients indicated that the nomogram may be widely and accurately used (17).

In the analysis of risk factors, age, pathological stage, CD4+ cell count and the method of surgical operation were independent risk factors related to prognosis. According to the nomogram, patients who had a lower pathological stage, $300-600 / \mathrm{mm}^{3} \mathrm{CD} 4+$ cell count, and underwent thoracotomy had a better prognosis and this result was similar to that indicated by a survival curve obtained by a Kaplan-Meier survival analysis.

In agreement with previous studies $(18,19)$, we confirmed age as an independent risk factor for the prognosis of lung cancer: the older the person is, the better the prognosis is; it is worse for younger patients. This may be due to a general decline in the ability of cells, particularly tumor cells, to divide in older patients, resulting in a decrease in the growth and invasiveness of tumors (20).

To our surprise, patients with CD4+ cell counts between $300 / \mathrm{mm}^{3}$ and $600 / \mathrm{mm}^{3}$ had a better prognosis. Generally speaking, CD4+ cells undertake many immune functions in the body, among which inhibiting tumor growth is a key 
Table 2 Multivariate analysis of the cohort

\begin{tabular}{|c|c|c|c|}
\hline Variable & \multicolumn{3}{|c|}{ Overall survival } \\
\hline \multicolumn{4}{|l|}{ Age } \\
\hline$<50$ & \multicolumn{3}{|c|}{ Intercept } \\
\hline$<60$ & 0.071 & 2.331 & $0.931-5.839$ \\
\hline \multicolumn{4}{|l|}{ CD4 cell count } \\
\hline$<300$ & \multicolumn{3}{|c|}{ Intercept } \\
\hline$<600$ & 0.019 & 2.801 & $1.179-6.653$ \\
\hline$<900$ & $<0.001$ & 16.796 & $3.495-80.715$ \\
\hline IA & \multicolumn{3}{|c|}{ Intercept } \\
\hline IB & 0.089 & 0.363 & $0.113-1.167$ \\
\hline$\| A$ & 0.748 & 0.694 & $0.074-6.463$ \\
\hline IIB & 0.034 & 0.143 & $0.023-0.868$ \\
\hline IIIA & 0.077 & 3.341 & $0.873-12.778$ \\
\hline IV & 0.706 & 0.671 & $0.084-5.360$ \\
\hline \multicolumn{4}{|l|}{ Surgery method } \\
\hline Thoracotomy & \multicolumn{3}{|c|}{ Intercept } \\
\hline
\end{tabular}

VATS, video-assisted thoracoscopic surgery.

function (21). Accordingly, the larger the CD4+ cell count, the stronger the expected tumor inhibitory activity and the better the deduced prognosis. The apparent discrepancy may be due to the presence in our study of only a small number of patients who had a CD4+ cell count of more than $900 / \mathrm{mm}^{3}$.

Most HIV-infected patients who develop lung cancer have a high probability of lymph node metastasis and VATS is generally recommended for early lung cancer. In our predictive map, the prognosis of patients who undergo VATS is poor (7). HIV-infection is often accompanied by a variety of opportunistic pulmonary infections and associated pleural and mediastinal lymph node adhesions. Such adhesions are a contraindication of VATS and accordingly a high proportion of our patients with early lung cancer underwent thoracotomy.

Although there are some shortcomings, the new prediction model can combine a variety of independent risk factors to predict the survival probability more accurately than a simple survival curve. Clinicians can use it to guide diagnosis and treatment. The procedures giving the highest probability of survival can then be selected for implementation. In fact, the prognosis of HIV-infected patients with lung cancer is generally poor and the issuance of relevant clinical diagnosis and treatment guidelines is a matter of urgency. In the interim, this new predictive model can provide guidance to improve the prognosis of patients as much as possible.

\section{Conclusions}

This study developed a novel nomogram with a moderate accuracy to help clinicians assess the prognosis of lung cancer patients with HIV infection who undergo surgery. This tool requires external validation, and additional studies 


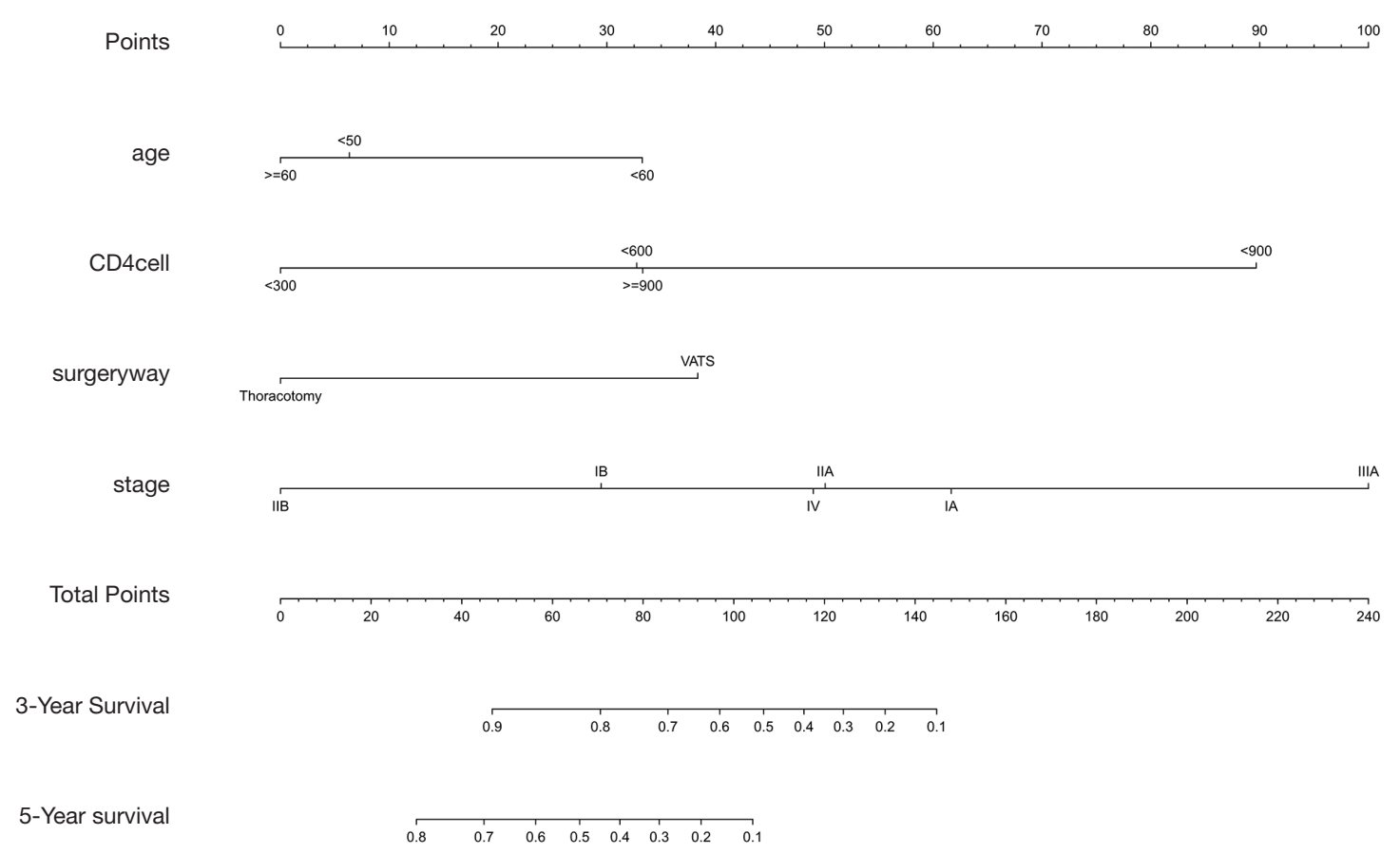

Figure 1 Developed prognosis nomogram. The prognostic nomogram was developed in the cohort from the variables of age, CD4+ cell count, surgery method, and pathology stage.
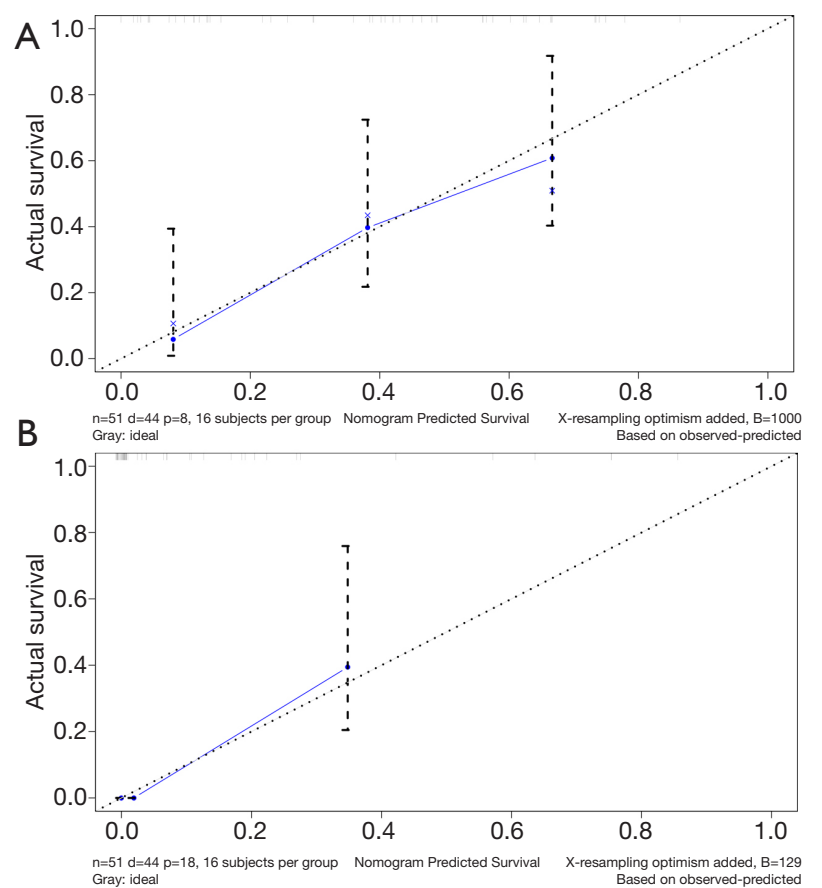

Figure 2 The calibration curves for predicting patient survival at (A) 3 years and (B) 5 years. The nomogram-predicted probability of overall survival is plotted on the $\mathrm{x}$-axis; the actual overall survival is plotted on the $y$-axis. are required to determine whether it can be applied to other patient groups.

\section{Acknowledgments}

Funding: None.

\section{Footnote}

Reporting Checklist: The authors have completed the TRIPOD reporting checklist. Available at http://dx.doi. org/10.21037/jtd-20-2268

Data Sharing Statement: Available at http://dx.doi. org/10.21037/jtd-20-2268

Conflicts of Interest: All authors have completed the ICMJE uniform disclosure form (available at http://dx.doi. org/10.21037/jtd-20-2268). The authors have no conflicts of interest to declare.

Ethical Statement: The authors are accountable for all aspects of the work in ensuring that questions related to the accuracy or integrity of any part of the work are appropriately investigated 
and resolved. The study was conducted in accordance with the Declaration of Helsinki (as revised in 2013). The study was approved by the Shanghai Public Health Clinical Center's ethics committee (Approval No. [2018] Y038) and informed consent was taken from all the patients.

Open Access Statement: This is an Open Access article distributed in accordance with the Creative Commons Attribution-NonCommercial-NoDerivs 4.0 International License (CC BY-NC-ND 4.0), which permits the noncommercial replication and distribution of the article with the strict proviso that no changes or edits are made and the original work is properly cited (including links to both the formal publication through the relevant DOI and the license). See: https://creativecommons.org/licenses/by-nc-nd/4.0/.

\section{References}

1. Hakimian R, Fang $\mathrm{H}$, Thomas $\mathrm{L}$, et al. Lung cancer in HIV-infected patients in the era of highly active antiretroviral therapy. J Thorac Oncol 2007;2:268-72.

2. Engels EA, Pfeiffer RM, Goedert JJ, et al. Trends in cancer risk among people with AIDS in the United States 1980-2002. AIDS 2006;20:1645-54.

3. Shiels MS, Pfeiffer RM, Gail MH, et al. Cancer burden in the HIV-infected population in the United States. J Natl Cancer Inst 2011;103:753-62.

4. Wada N, Jacobson LP, Cohen M, et al. Cause-specific life expectancies after 35 years of age for human immunodeficiency syndrome-infected and human immunodeficiency syndrome-negative individuals followed simultaneously in long-term cohort studies, 1984-2008. Am J Epidemiol 2013;177:116-25.

5. Patel P, Hanson DL, Sullivan PS, et al. Incidence of types of cancer among HIV-infected persons compared with the general population in the United States, 1992-2003. Ann Intern Med 2008;148:728-36.

6. Deeken JF, Tjen-A-Looi A, Rudek MA, et al. The rising challenge of non-AIDS-defining cancers in HIV-infected patients. Clin Infect Dis 2012;55:1228-35.

7. Boffa DJ, Kosinski AS, Furnary AP, et al. Minimally Invasive Lung Cancer Surgery Performed by Thoracic Surgeons as Effective as Thoracotomy. J Clin Oncol 2018;36:2378-85.

8. Solomon SS, Solomon S, McFall AM, et al. Integrated HIV testing, prevention, and treatment intervention for key populations in India: a cluster-randomised trial. Lancet HIV 2019;6:e283-96.

9. Mao Q, Xia W, Dong G, et al. A nomogram to predict the survival of stage IIIA-N2 non-small cell lung cancer after surgery. J Thorac Cardiovasc Surg 2018;155:1784-92.e3.

10. Wo Y, Yang H, Zhang Y, et al. Development and External Validation of a Nomogram for Predicting Survival in Patients With Stage IA Non-small Cell Lung Cancer $\leq 2$ cm Undergoing Sublobectomy. Front Oncol 2019;9:1385.

11. Wu J, Shen Z. Exosomal miRNAs as biomarkers for diagnostic and prognostic in lung cancer. Cancer Med. 2020;9:6909-22.

12. Pencina MJ, D'Agostino RB. Overall $\mathrm{C}$ as a measure of discrimination in survival analysis: model specific population value and confidence interval estimation. Stat Med 2004;23:2109-23.

13. Iasonos A, Schrag D, Raj GV, et al. How to build and interpret a nomogram for cancer prognosis. J Clin Oncol 2008;26:1364-70.

14. Shi H, Wang XH, Gu JW, et al. Development and Validation of Nomograms for Predicting the Prognosis of Triple-Negative Breast Cancer Patients Based on 379 Chinese Patients. Cancer Manag Res 2019;11:10827-39.

15. Gittleman H, Sloan AE, Barnholtz-Sloan JS. An independently validated survival nomogram for lowergrade glioma. Neuro Oncol 2020;22:665-74.

16. Wang S, Yang L, Ci B, et al. Development and Validation of a Nomogram Prognostic Model for SCLC Patients. J Thorac Oncol 2018;13:1338-48.

17. Khalife H, Skafi N, Fayyad-Kazan M, et al. MicroRNAs in breast cancer: New maestros defining the melody. Cancer Genet 2020;246-247:18-40.

18. Kirk GD, Merlo C, O' Driscoll P, et al. HIV infection is associated with an increased risk for lung cancer, independent of smoking. Clin Infect Dis 2007;45:103-10.

19. Sigel K, Wisnivesky J, Gordon K, et al. HIV as an independent risk factor for incident lung cancer. AIDS 2012;26:1017-25.

20. Yin HL, Xu HW, Lin QY. miR129-1 regulates protein phosphatase 1D protein expression under hypoxic conditions in non-small cell lung cancer cells harboring a TP53 mutation. Oncol Lett 2020;20:2239-47.

21. Pan Y, Jin K, Xie X, et al. MicroRNA-19a-3p inhibits the cellular proliferation and invasion of non-small cell lung cancer by downregulating UBAP2L. Exp Ther Med 2020;20:2252-61.

Cite this article as: Wang L, Wu L, Liu J, Wan L, Chen H, Zhu Y, Wang J, Li H, Shi L, Li L, Song Y. Prognostic nomogram for surgery of lung cancer in HIV-infected patients. J Thorac Dis 2021;13(1):76-81. doi: 10.21037/jtd-20-2268 